\title{
Calibration of low-temperature ac susceptometers with a copper cylinder standard
}

\author{
D.-X. Chen and V. Skumryev \\ ICREA and Departament de Física, Universitat Autònoma de Barcelona, Bellaterra, 08193 Barcelona, Spain
}

(Received 4 November 2009; accepted 15 January 2010; published online 18 February 2010)

\begin{abstract}
A high-quality low-temperature ac susceptometer is calibrated by comparing the measured ac susceptibility of a copper cylinder with its eddy-current ac susceptibility accurately calculated. Different from conventional calibration techniques that compare the measured results with the known property of a standard sample at certain fixed temperature $T$, field amplitude $H_{m}$, and frequency $f$, to get a magnitude correction factor, here, the electromagnetic properties of the copper cylinder are unknown and are determined during the calibration of the ac susceptometer in the entire $T, H_{m}$, and $f$ range. It is shown that the maximum magnitude error and the maximum phase error of the susceptometer are less than $0.7 \%$ and $0.3^{\circ}$, respectively, in the region $T=5-300 \mathrm{~K}$ and $f$ $=111-1111 \mathrm{~Hz}$ at $H_{m}=800 \mathrm{~A} / \mathrm{m}$, after a magnitude correction by a constant factor as done in a conventional calibration. However, the magnitude and phase errors can reach $2 \%$ and $4.3^{\circ}$ at 10000 and $11 \mathrm{~Hz}$, respectively. Since the errors are reproducible, a large portion of them may be further corrected after a calibration, the procedure for which is given. Conceptual discussions concerning the error sources, comparison with other calibration methods, and applications of ac susceptibility techniques are presented. () 2010 American Institute of Physics. [doi:10.1063/1.3309779]
\end{abstract}

\section{INTRODUCTION}

Although low-temperature ac susceptometers have been fabricated in a number of companies and widely used in materials research, ${ }^{1-5}$ their calibration has been less studied. Few researchers emphasize the accuracy of their measured susceptibilities, and when a negative imaginary susceptibility is obtained, the correctness of the measured results is the first question to be asked and is often not answered. ${ }^{6-10}$ We study this topic in the present work.

Using an ac susceptometer, the sample is placed within an assembly of magnetizing, measuring, and compensating coils, which are coaxially or both coaxially and concentrically configurated, ${ }^{11,12}$ the ac field $H(t)=H_{m} \sin (2 \pi f t)$ is produced by the magnetizing coil and determined by the current flowing through it, and the ac magnetic moment is determined by the induced electromagnetic force (emf) in the measuring and compensating coils. A lock-in amplifier is usually used for providing the ac signal, which is amplified to feed the magnetizing coil, and for measuring the magnetizing current (through the voltage across a standard resistor) and the induced emf. With well designed and accurately manufactured coil formers and uniformly wound coils of known numbers of turns and with perfect air-flux compensation when no sample is present, the field constant (the field produced by a unit magnetizing current) and moment sensitivity (the emf induced by a unit moment and a unit $f$ ) can ideally be known with high precision. In reality, however, the emf in the counterwound measuring and compensating coils when a sample is absent cannot be exactly zero, so that the measurement should be done iteratively with and without sample (or situating the sample iteratively in the measuring and compensating coils for the coaxial configuration) to make an empty coil correction. The magnetizing coil is relatively large with fewer number of turns and easy to be made precisely, so that the field constant can be determined with high accuracy. For smaller measuring and compensating coils with large numbers of turns, it is very difficult to get a moment sensitivity with high accuracy, so a calibration of the moment sensitivity is always necessary after the coil assembly is made. Such a calibration becomes unavoidable if there is a superconducting magnet surrounding the ac coil assembly, for which the moment sensitivity cannot be calculated accurately. Conventionally, the calibration is carried out by measuring a standard sample with known susceptibility at room temperature and certain values of $H_{m}$ and $f$.

In the present work, we calibrate a low-temperature ac susceptometer with a copper cylinder as the standard sample. Different from the conventional calibration using a standard sample with known (mass) susceptibility, the susceptibility of the copper cylinder is unknown before calibration; it is determined together with the moment sensitivity of the ac susceptometer during the calibration. The calibration is done not only at room temperature, at which the susceptibility of the usual standard sample is known, but in the entire $T, H_{m}$, and $f$ range, so that the performance of the susceptometer can be characterized completely.

The sample and susceptometer are explained in Sec. II. The calibration is described in Sec. III. The correction method after calibration is described in Sec. IV. Discussions relating to the errors, calibration, and applications of ac susceptibility measurements are presented in Sec. V.

\section{SAMPLE AND SUSCEPTOMETER}

The standard sample was a copper cylinder, whose diameter $2 a$ and length $2 l$ were machined to be $5 \mathrm{~mm}$, with volume $V=9.82 \times 10^{-8} \mathrm{~m}^{3}$. The studied ac susceptometer was the ac measurement system (ACMS) of Quantum Design's physical property measurement system (PPMS). ${ }^{13}$ 
This susceptometer has a coil assembly of typical coaxial configuration. The copper drive (magnetizing) coil of about $10 \mathrm{~cm}$ in length is wound longitudinally around a pair of counterwound identical top and bottom (measuring and compensating) copper detection coils connected in series and separated by several centimeters. During measurements at a fixed ac field, the sample is situated iteratively at the center of each detection coil to get two readings. The empty coil contribution to the moment measurements is removed by subtracting both readings.

Different from the general coil arrangement described in Sec. I, there is an extra pair of calibration coils and an extra magnetizing moment compensating coil, by which the performance of the susceptometer is improved. Surrounding the ac coils, there is a dc superconducting magnet always immersed in liquid helium.

The calibration coils are single-turn coils connected in series and are situated at the center of each detection coil, where the sample measurements occur. After sample moment measurements at a fixed ac field, the sample is placed at the center of the coil assembly (symmetrically between both calibration coils) and two readings are taken with the calibration coils switched into the detection circuit with opposing polarities. The calibration readings are subtracted to remove the contribution of the sample according to the instruction manual (we will comment on this in Sec. IV), so that a calibration vector in the complex plane is obtained. This vector is used for the phase correction of the magnetizing field.

The magnetizing moment compensation coil is counterwound surrounding the drive coil and series connected to it, such that the total magnetic moment of both coils becomes zero. In this case, while producing the ac magnetizing field in the sample region, the magnetizing current results in very small fields in the surrounding volume, where several metallic tubes for the cryogenic system and the superconducting magnet exist. Thus, the normal or superconducting eddy currents in the surrounding conducting or superconducting parts induced by the ac flux produced by the magnetizing current will be much reduced, so that the ac field may be calculated from the magnetizing current itself.

A bias dc field up to $9 \mathrm{~T}$ can be produced by the superconducting magnet. During ac measurement, the dc circuit is always closed even if the dc field is set to zero.

\section{CALIBRATION}

\section{A. Measurement results}

The complex ac susceptibility $\chi=\chi^{\prime}-j \chi^{\prime \prime}$ of the copper cylinder was measured using the incorporated software at zero dc field as a function of ac field amplitude $H_{m}$, frequency $f$, and temperature $T$, following a standard procedure after the sample was automatically centered. Writing the measured complex moment amplitude as $m=m^{\prime}-j m^{\prime \prime}, \chi$ is calculated in SI units as

$$
\chi^{\prime}=k m^{\prime} /\left(H_{m} V\right),
$$

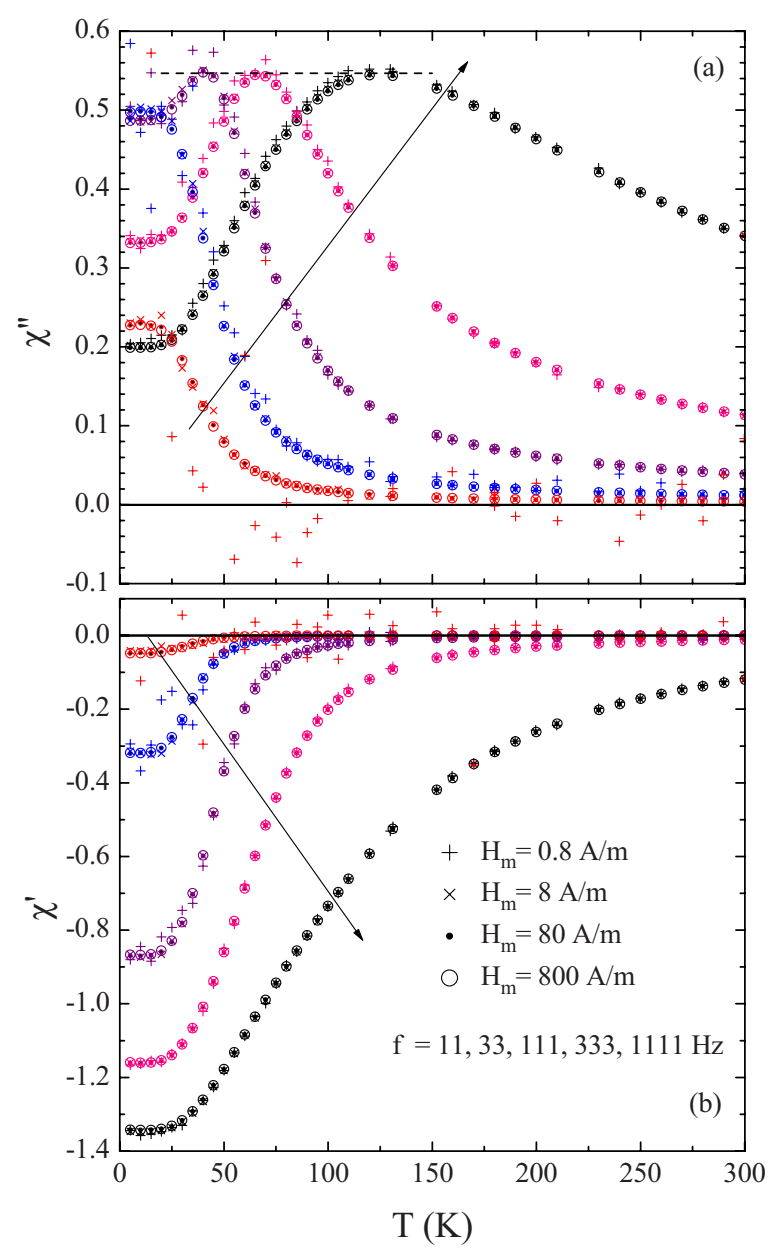

FIG. 1. (Color online) Measured susceptibility $\chi=\chi^{\prime}-j \chi^{\prime \prime}$ of copper cylinder as a function of ac field amplitude $H_{m}$, frequency $f$, and temperature $T$. Arrows indicate the direction of increasing $f$.

$$
\chi^{\prime \prime}=k m^{\prime \prime} /\left(H_{m} V\right),
$$

where $k$ is a factor for magnitude correction. $k=1$ occurs if no correction is made. The frequency dependence of the induced emf is accounted for in $m^{\prime}$ and $m^{\prime \prime}$.

The $\chi^{\prime \prime}$ and $\chi^{\prime}$ of the sample measured at $H_{m}=0.8,8,80$, and $800 \mathrm{~A} / \mathrm{m}$ and $f=11,33,111,333$, and $1111 \mathrm{~Hz}$, and calculated by Eqs. (1) and (2) with $k=1.081$ (see below), as functions of $T$ are plotted in Figs. 1(a) and 1(b).

We see that there are significant data scattering at low $f$ and low $H_{m}$. The scattering becomes negligible at high $H_{m}$, and $\chi^{\prime}(f, T)$ and $\chi^{\prime \prime}(f, T)$ measured at $H_{m}=80$ and $800 \mathrm{~A} / \mathrm{m}$ coincide well to each other. Thus, we use for calibration the measured data at $H_{m}=800 \mathrm{~A} / \mathrm{m}$, where the smallest random error is expected.

The ac moment of the copper cylinder arises from its inner eddy currents induced by the applied ac field. Since the conductivity $\sigma$ of copper at a fixed $T$ is independent of low magnetic field, the coincidence in the measured $\chi(f, T)$ between $H_{m}=80$ and $800 \mathrm{~A} / \mathrm{m}$ is expected. Since $\sigma$ increases with decreasing $T$, the effect of decreasing $T$ is qualitatively the same as increasing $f$. Therefore, we have in Fig. 1, with decreasing $T$ and increasing $f, \chi^{\prime}$ decreases and $\chi^{\prime \prime}$ increases and then decreases, showing the same maximum value of $\chi_{m}^{\prime \prime}$ for $f=111,333$, and $1111 \mathrm{~Hz}$. 


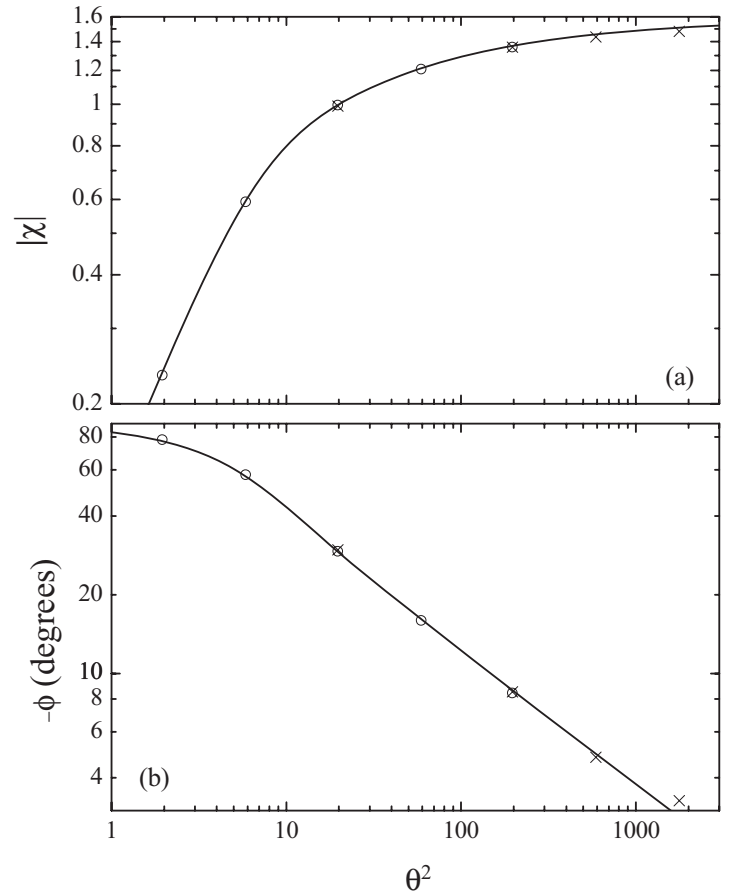

FIG. 2. The theoretical $|\chi|$ and $-\phi$ of a conducting cylinder of aspect ratio $\gamma=1$ as functions of dimensionless frequency $\theta^{2}$ (lines) compared with those converted from the $\chi$ of copper cylinder measured at $T=10 \mathrm{~K}$ with $H_{m}$ $=800 \mathrm{~A} / \mathrm{m}$ and $f=11,33,111,333$, and $1111 \mathrm{~Hz}$ (circles) and with $H_{m}$ $=8 \mathrm{~A} / \mathrm{m}$ and $f=111,1111,3333$, and $10000 \mathrm{~Hz}$ (crosses).

\section{B. Magnitude correction factor}

The magnitude correction factor $k$ and conductivity $\sigma$ are determined by comparing the measured $\chi(f)$ of the copper cylinder with the theoretical $\chi$ of a nonmagnetic conducting cylinder of $\gamma \equiv l / a=1$, given in Ref. 14. Since a maximum $\sigma$ occurs at the lowest $T$ and $\chi$ is stable at $T \leq 15 \mathrm{~K}$, we use the measured $\chi^{\prime}(f)$ and $\chi^{\prime \prime}(f)$ at $H_{m}=800 \mathrm{~A} / \mathrm{m}$ and $T=10 \mathrm{~K}$ to make the magnitude correction. It is necessary to convert $\chi^{\prime}$ and $\chi^{\prime \prime}$ into the magnitude susceptibility $|\chi|$ and phase $\phi$ as

$$
\begin{aligned}
& |\chi|=\sqrt{\chi^{\prime 2}+\chi^{\prime \prime 2}}, \\
& \phi=\arctan \left(\chi^{\prime \prime} / \chi^{\prime}\right),
\end{aligned}
$$

where arctangent takes its principal value, i.e., $-\pi / 2 \leq \phi$ $\leq \pi / 2$. In eddy-current ac susceptibility calculations, the volume averaged susceptibility $\chi$ for different values of $\gamma$ is given as a function of the dimensionless frequency ${ }^{14}$

$$
\theta^{2}=2 \pi f \mu_{0} \sigma a^{2} .
$$

Converted from the data $\chi^{\prime}\left(\theta^{2}\right)$ and $\chi^{\prime \prime}\left(\theta^{2}\right)$ in Tables I and II of Ref. 14 for $\gamma=1$ using Eqs. (3) and (4), the theoretical $|\chi|\left(\theta^{2}\right)$ and $-\phi\left(\theta^{2}\right)$ functions are plotted by solid spline curves in Figs. 2(a) and 2(b), respectively.

Converting the measured $\chi^{\prime}(f)$ and $\chi^{\prime \prime}(f)$ at $T=10 \mathrm{~K}$ and $f=11,33,111,333$, and $1111 \mathrm{~Hz}$ into $|\chi|\left(\theta^{2}\right)$ and $-\phi\left(\theta^{2}\right)$ using Eqs. (1)-(5) by assuming certain values of $k$ and $\sigma$, five experimental points are plotted in each of Figs. 2(a) and 2(b) by open circles. We adjust iteratively the values of $k$ and $\sigma$ until the best agreement between the theoretical and experimental results is reached in Fig. 2(a), so that $k=1.081$ and $\sigma=3.423 \times 10^{9} \mathrm{~S} / \mathrm{m}($ at $10 \mathrm{~K})$ are finally determined.

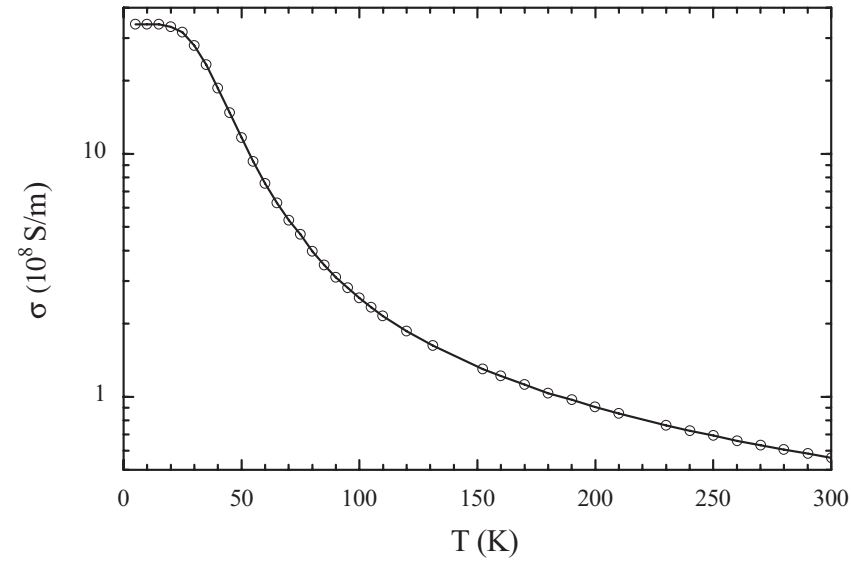

FIG. 3. The conductivity $\sigma$ of copper as a function of temperature $T$, determined from the measured $|\chi|(T)$ of the cylinder at $H_{m}=800 \mathrm{~A} / \mathrm{m}$ and $f$ $=333 \mathrm{~Hz}$.

\section{Determination of conductivity versus temperature}

After $k$ is determined, $\sigma$ may be in principle determined as a function of temperature by comparing the measured $|\chi|(T)$ or $-\phi(T)$ at $H_{m}=800 \mathrm{~A} / \mathrm{m}$ and any value of $f$ with theoretical $|\chi|\left(\theta^{2}\right)$ or $-\phi\left(\theta^{2}\right)$. It is better to use $|\chi|(T)$, whose error is in general smaller than that of $-\phi(T)$. Examining the data in Fig. 1, we see that the data scattering for $f=333$ and $1111 \mathrm{~Hz}$ is the smallest. Since systematic error should increase with increasing $f$, we choose $f=333 \mathrm{~Hz}$ to determine $\sigma(T)$.

We draw a theoretical $|\chi|\left(\theta^{2}\right)$ curve like in Fig. 2(a) and compare it point by point with the measured $|\chi|(T)$, so that $\theta^{2}$ as a function of $T$ is numerically obtained. With $\theta^{2}(T)$ and $f=333 \mathrm{~Hz}$, the final $\sigma(T)$ is calculated using Eq. (5), as shown in Fig. 3.

\section{Error in susceptibility}

Having $\sigma(T)$, the measured $\chi(T)$ at any given $f$ may be converted into experimental $\chi\left(\theta^{2}\right)$ using Eq. (5). The comparison between the experimental $|\chi|\left(\theta^{2}\right)$ and $-\phi\left(\theta^{2}\right)$ and the theoretical ones is shown in Figs. 4 and 5, respectively. We see from Fig. 4 that with respect to $|\chi|$, the agreement between the experimental and theoretical results is good with maximum difference of $0.8 \%$ for $f$ between 11 and $1111 \mathrm{~Hz}$, corresponding to $\theta^{2}$ between 0.03 and 200. With respect to $\phi$, however, a good agreement within $\pm 0.3^{\circ}$ occurs for $f$ $\geq 111 \mathrm{~Hz}$, and there is a negative error at $f=33 \mathrm{~Hz}$ down to $-0.6^{\circ}$ in low- $T$ (high $\theta^{2}$ ) region and a positive error at $f$ $=11 \mathrm{~Hz}$ up to $4.3^{\circ}$ in high- $T$ region.

For higher $f$, lower $H_{m}$ has to be used to avoid overloading the detector. We have repeated the above measurements at $H_{m}=8 \mathrm{~A} / \mathrm{m}$ and $f=111,1111,3333$, and $10000 \mathrm{~Hz}$, and the comparison between experimental and theoretical results, based on the $\sigma(T)$ determined above, is shown in Figs. 6 and 7. Compared with Figs. 4 and 5 for $H_{m}=800 \mathrm{~A} / \mathrm{m}$, the agreement for $f=111$ and $1111 \mathrm{~Hz}$ is slightly worse in Figs. 6 and 7 for $H_{m}=8 \mathrm{~A} / \mathrm{m}$. We see in Figs. 6(c) and 6(d) that a systematic negative error, down to $-1 \%$ and $-2 \%$, occurs for $f=3333$ and $10000 \mathrm{~Hz}$, respectively. The situation for $\phi$ shown in Fig. 7 is more complicated. There is a small posi- 

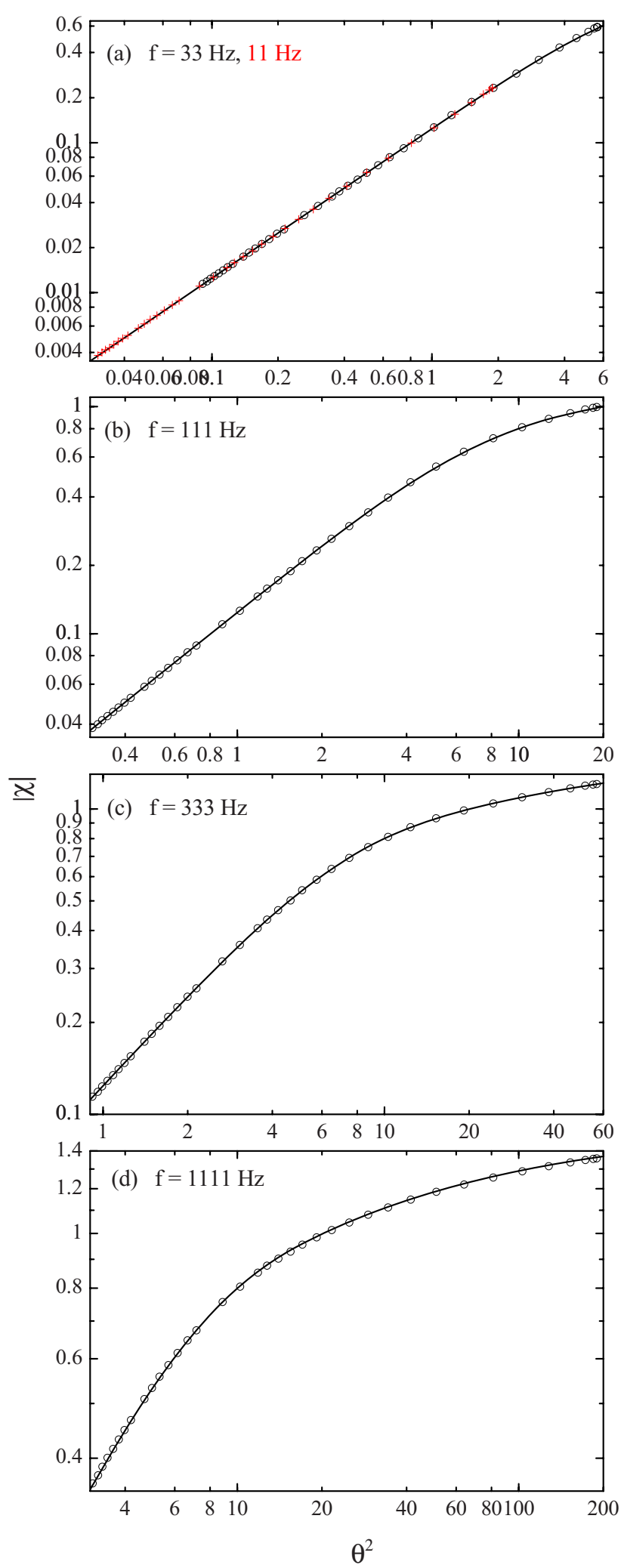

FIG. 4. (Color online) The theoretical $|\chi|$ of a conducting cylinder of $\gamma=1$ as a function of $\theta^{2}$ (lines) compared with that converted from the $\chi$ of the copper cylinder measured at $T=5$ to $300 \mathrm{~K}$ with $H_{m}=800 \mathrm{~A} / \mathrm{m}$ and $f=11$ (crosses), 33, 111, 333, and $1111 \mathrm{~Hz}$ (circles).

tive error up to $0.2^{\circ}$ at low $T$ for $f=3333 \mathrm{~Hz}$ but the positive error for $f=10000 \mathrm{~Hz}$ changes into negative at $T=25 \mathrm{~K}$ and decreases down to $-0.5^{\circ}$. However, both errors are negligible at $f=10000 \mathrm{~Hz}$ if $T>175 \mathrm{~K}$.

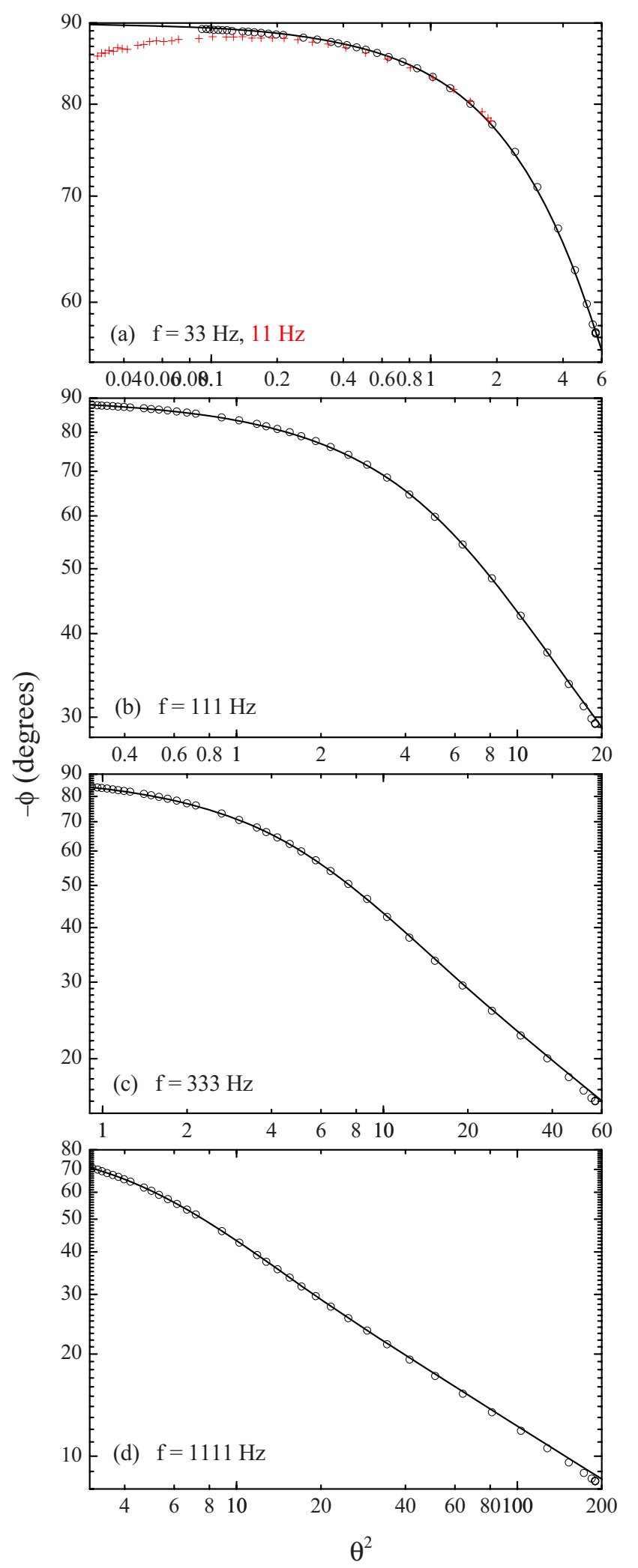

FIG. 5. (Color online) The theoretical $-\phi$ of a conducting cylinder of $\gamma$ $=1$ as a function of $\theta^{2}$ (lines) compared with that converted from the $\chi$ of the copper cylinder measured at $T=5$ to $300 \mathrm{~K}$ with $H_{m}=800 \mathrm{~A} / \mathrm{m}$ and $f$ $=11$ (crosses), 33, 111, 333, and $1111 \mathrm{~Hz}$ (circles).

\section{MAGNITUDE AND PHASE CORRECTIONS}

By the above calibration, we see that the performance of the studied ac susceptometer is excellent after a magnitude correction with constant $k=1.081$. Even so, its performance 

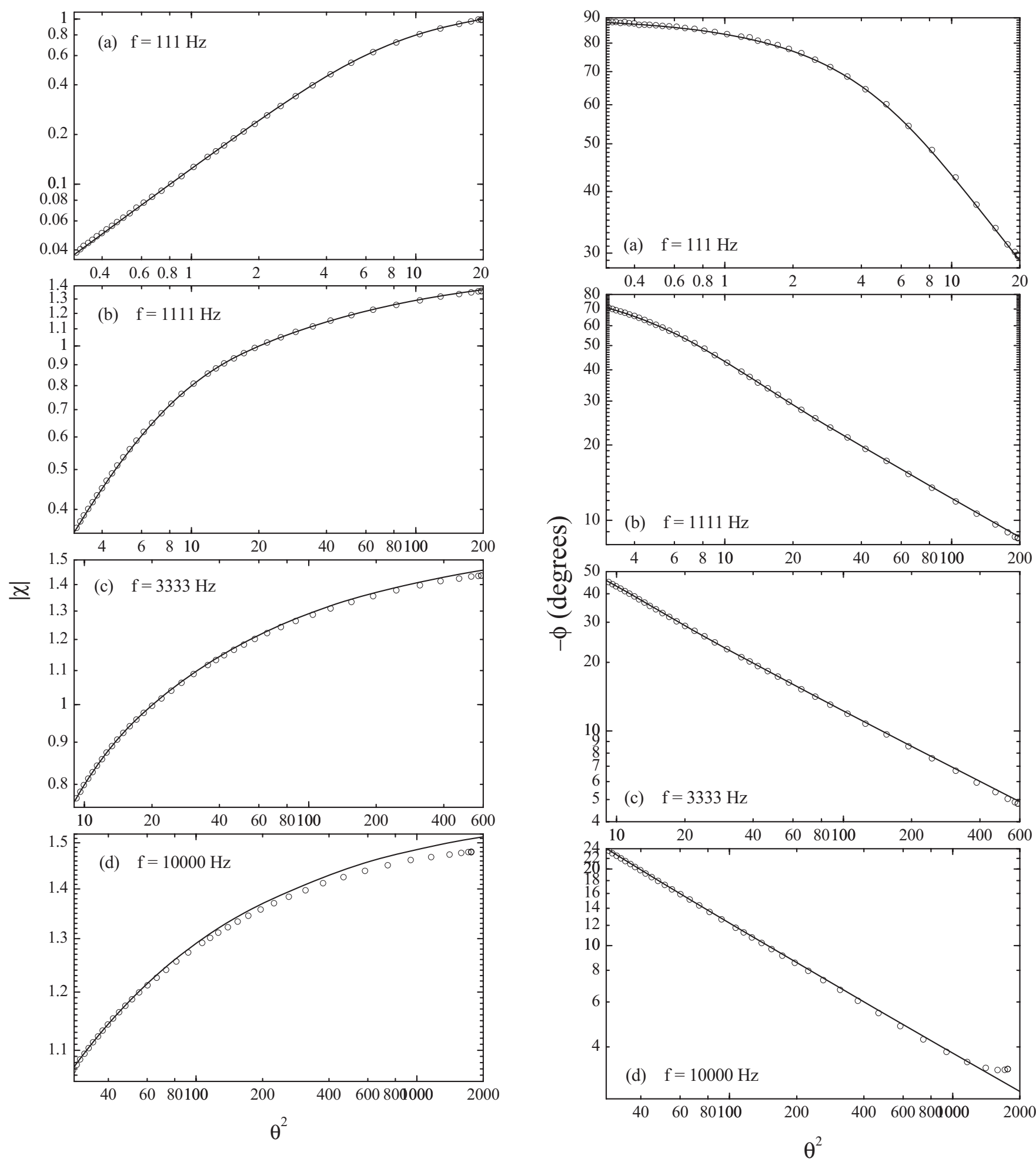

FIG. 6. Same as Fig. 4 but with $H_{m}=8 \mathrm{~A} / \mathrm{m}$ and $f=111,1111,3333$, and $10000 \mathrm{~Hz}$.

can be further improved by a $f$ and $T$ dependent correction, if the error is proven to be systematic and stable.

Assuming the field is accurate and all the error comes from the measured complex magnetic moment, whose amplitude is expressed by

$$
m=m^{\prime}-j m^{\prime \prime}=|m| e^{-j \phi},
$$

the correction should be done for $m$. In Eq. (6), $|m|$ and $\phi$ are the magnitude and phase of $m$, whose relations to $m^{\prime}$ and $m^{\prime \prime}$ are

FIG. 7. Same as Fig. 5 but with $H_{m}=8 \mathrm{~A} / \mathrm{m}$ and $f=111,1111,3333$, and $10000 \mathrm{~Hz}$.

$$
\begin{aligned}
& |m|=\sqrt{m^{\prime 2}+m^{\prime 2}}, \\
& \phi=\arctan \left(m^{\prime \prime} / m^{\prime}\right),
\end{aligned}
$$

where arctangent takes its principal value, i.e., $-\pi / 2 \leq \phi$ $\leq \pi / 2$, and $m^{\prime}$ and $m^{\prime \prime}$ are calculated from $|m|$ and $\phi$ by

$$
m^{\prime}=|m| \cos \phi,
$$




$$
m^{\prime \prime}=|m| \sin \phi,
$$

if $m^{\prime}>0$, and by

$$
\begin{aligned}
& m^{\prime}=|m| \cos (\phi+\pi), \\
& m^{\prime \prime}=|m| \sin (\phi+\pi),
\end{aligned}
$$

if $m^{\prime}<0$.

The magnitude correction changes $|m|$ into $k(f, T)|m|$, whereas the phase correction changes $\phi$ into $\phi+\Delta \phi(f, T)$. Both $k(f, T)$ and $\Delta \phi(f, T)$ are functions of $f$ and $T$. As a result, after the correction, Eqs. (1) and (2) are replaced by

$$
\begin{aligned}
\chi^{\prime} & =\frac{k(f, T)|m| \cos [\phi+\Delta \phi(f, T)]}{H_{m} V}, \\
\chi^{\prime \prime} & =\frac{k(f, T)|m| \sin [\phi+\Delta \phi(f, T)]}{H_{m} V},
\end{aligned}
$$

if $m^{\prime}>0$ (after correction), and by

$$
\begin{aligned}
& \chi^{\prime}=\frac{k(f, T)|m| \cos [\phi+\Delta \phi(f, T)+\pi]}{H_{m} V}, \\
& \chi^{\prime \prime}=\frac{k(f, T)|m| \sin [\phi+\Delta \phi(f, T)+\pi]}{H_{m} V},
\end{aligned}
$$

if $m^{\prime}<0$ (after correction).

We have stressed the condition "after correction" since in some case the correction may change the sign of $m^{\prime}$. Using the subscripts "exp" and "theo" to stand for the experimental and theoretical values, $k(f, T)$ and $\Delta \phi(f, T)$ are defined as $m_{m \text {,theo }}(f, T) / m_{m \text {,exp }}(f, T)$ and $\phi_{\text {theo }}(f, T)-\phi_{\text {exp }}(f, T)$, respectively. We note that each data point in Figs. 4-7 corresponds to a value of $T$, although at different $f$, the same value of $T$ corresponds to different value of $\theta^{2}$.

\section{DISCUSSION}

\section{A. Error sources and corrections}

The electromagnetic properties of the magnetizing field coil may be studied by its equivalent circuit consisting of a parallel connection of its distribution capacitance $C$ and its dc inductance $L$ and dc resistance $R$, both connected in series. The current flowing through the coil is divided into a displacement current flowing through $C$ with a $90^{\circ}$ leading phase and a magnetizing current flowing through $L$ and $R$ with a certain phase delay, so that an error in the ac field will occur when using the measured total current to stand for the magnetizing current. The error in field measurements owing to this is negative in magnitude and positive in phase, and it can be significant for big magnetizing coils of high-field ac susceptometers, ${ }^{12}$ for which both $L$ and $C$ are large, at high $f$ and low $T$. For the studied susceptometer, however, this error is practically negligible since the coil is small. The dominant error for the field measurements could arise from the ferromagnetic, conducting, or superconducting parts inside or around the coil.

The ac field produced by the applied current magnetizes the ferromagnets so that magnetic poles appear in them, and the field produced by these poles in the sample region forms an error in ac field. This error is not simply proportional to the applied ac current owing to the history dependence of ferromagnetism, so that the way to remove this error is to position all the ferromagnetic parts far away from the coil. This is done for all properly manufactured ac susceptometers.

The existence of conducting (and superconducting in the present case) parts is unavoidable. There are the stainless steel/copper sample tube, inner and outer stainless steel vacuum tubes, and a superconducting magnet surrounding the ac coils in the metallic Dewar for the studied susceptometer. Eddy currents are induced by the applied ac flux in all the conducting parts and the eddy currents produce fields in the sample region, resulting in magnitude and phase errors in field measurements. In the studied ac susceptometer, the inclusion of the magnetizing moment-compensating coil is important for reducing such errors. The error in phase is corrected by setting the pair of calibration coils and two extra measurements. According to Ref. 13, subtracting the two readings with opposite coil polarities may cancel the contribution of the sample, but we have different opinion on this. The moment of the sample is induced by and proportional to the ac field, so that the flux linked to the calibration coils always include both contributions from the field and the sample moment, and one cannot remove the contribution of the sample moment alone by subtracting two readings. Therefore, although the phase error of the field can be effectively reduced by this calibration, there is still an uncertainty remaining from the interference of the sample moment.

The dominant error for the moment measurements comes from the eddy currents in the conducting parts near the sample. The ac fields produced by the ac moment of the sample itself induce eddy currents in the surrounding conducting parts, and the induced eddy currents produce an extra flux linked to the detection coils so that an error in moment measurements occurs. This error increases with increasing $f$ and decreasing $T$, i.e., increasing the conductivity of the conducting parts. It is correctable by a factor $k(f, T) \exp [-j \Delta \phi(f, T)]$ since eddy currents are always proportional to the ac moment of the sample. The error in the above susceptibility measurements of the studied susceptometer should mainly come from this.

There is another error source coming from the lock-in amplifier, or digital signal processor in the studied ac susceptometer, for which the frequency is most accurately set, the measurements of voltage magnitude can be very accurate, but the phase error may be significant at certain values of frequency.

\section{B. Basis of calibration with a copper cylinder}

The magnetic properties of conventional working standard samples are transmitted from a primary standard sample made of standard reference material of high purity, whose paramagnetic mass susceptibility has been accurately determined by absolute measurements at certain values of $T .{ }^{15} \mathrm{In}$ our calibration, the relevant electromagnetic properties of the copper cylinder are originally unknown and are determined together with the calibration of the ac susceptometer, based on basic laws and accurate measurements. In other words, 
both the standard sample and ac susceptometer are mutually calibrated, although after such a first calibration, the standard sample can be used independently.

The calibration is realized based on the following assumptions: (i) the diameter $2 a$ and length $2 l$ of the copper cylinder are machined accurately, (ii) its conductivity $\sigma$ is constant when temperature is set at $T_{1}$, (iii) the measurement frequency $f$ is accurate, (iv) the systematic error in $|\chi|$ is independent of $f$ in an enough-large $f$ range, so that it can be corrected by a factor $k$, and (v) the theoretical $\chi\left(\theta^{2}\right)$ of conducting cylinders is accurate. The determined magnitude correction factor $k$ and the conductivity $\sigma\left(T_{1}\right)$ will be accurate if all these conditions are met. These assumptions are analyzed as follows.

For (i), although $2 a=2 l=5 \mathrm{~mm}$ has been checked during sample preparation by careful measurements, there is an uncertainty on the order of $1 \%$ for the volume $V$, which sets an upper limit of the accuracy of $k$ according to Eqs. (1) and (2). This error may be further corrected by another sample with better size accuracy, as described below. For (ii), a constant $\sigma$ can be well kept during measurements at $T_{1}=10 \mathrm{~K}$, and there is a negligible magnetoresistance effect at fields less than $800 \mathrm{~A} / \mathrm{m}$, so that no error results from a $\sigma$ variation. For (iii), the accuracy of $f$ for any good digital instrument is much better than $0.1 \%$, and it can be regarded as exact.

For (iv), the $f$ range may be chosen as follows. Owing to the eddy-current nature of the moment measurement error, the magnitude and phase errors are proportional to $f^{2}$ and $f$, respectively. Thus, there should be a low $f$ region, where the magnitude error is of second order. The width of the $f$ range is determined by the intensity of disturbing eddy currents induced in surrounding conducting parts, and it is below $1111 \mathrm{~Hz}$ for the present case. The systematic error in voltage measurement should be on the order of $0.1 \%$, which has a negligible contribution to the systematic error of $|\chi|$.

For (v), $\chi\left(\theta^{2}\right)$ of conducting cylinders is numerically calculated and tabulated in Ref. 14 based on the Maxwell equations and Ohm's law. Its accuracy is estimated to be on the order of $0.1 \%$ by comparing the results with the magnetometric demagnetizing factor of completely shielded cylinders numerically calculated in Refs. 16 and 17 and the exact $\chi\left(\theta^{2}\right)$ for conducting spheres derived in Ref. 18. Thus, the accuracy of theoretical $\chi\left(\theta^{2}\right)$ curve may be set as $0.2 \%$.

The above analysis is verified by the maximum deviation of experimental data points from the theoretical curve in Fig. 2(a) to be $0.15 \%$ at $f$ between 11 and $1111 \mathrm{~Hz}$, and it increases to $1 \%$ and $2 \%$ when $f=3333$ and $10000 \mathrm{~Hz}$.

In principle, $\sigma(T)$ may be obtained at any $f$ between 11 and $1111 \mathrm{~Hz}$. But since the sensitivity is proportional to $f$, we choose quite arbitrarily to use $333 \mathrm{~Hz}$. In our experience, for any ac susceptometer, the error owing to eddy currents and instrument electronics is the smallest at 111 and $333 \mathrm{~Hz}$ among the frequencies used in the present work.

\section{Calibration with other standard samples}

The copper cylinder as a nonmagnetic conducting standard sample can only be used in ac field, which induces eddy currents resulting in a magnetic moment of the sample. A round or square epitaxial $\mathrm{YBa}_{2} \mathrm{Cu}_{3} \mathrm{O}_{7-\delta}$ superconducting film

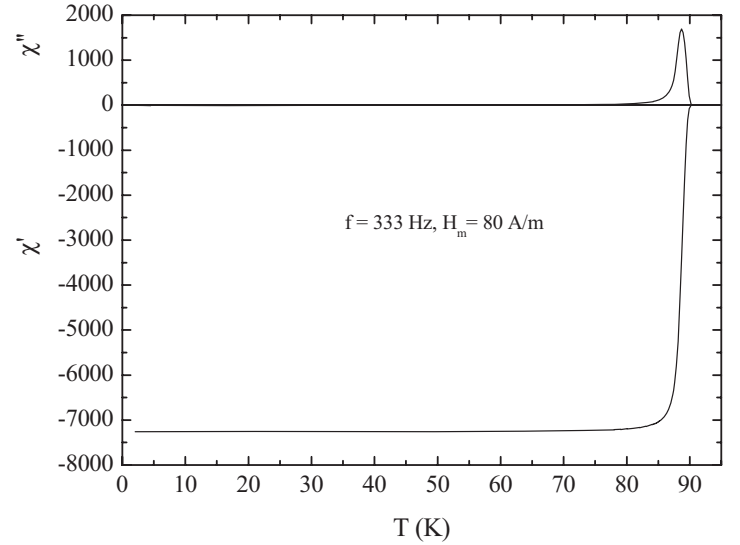

FIG. 8. The measured ac susceptibility of the studied square $\mathrm{YBa}_{2} \mathrm{Cu}_{3} \mathrm{O}_{7-\delta}$ superconducting film at $H_{m}=80 \mathrm{~A} / \mathrm{m}$ and $f=333 \mathrm{~Hz}$ as a function of $T$.

on a $\mathrm{LaAlO}_{3}$ single crystal substrate may be used as a standard sample in both the ac and dc cases but only at low temperatures and low fields. The geometry of the film may be perfectly prepared by photolithography and accurately measured by optical microscopy. When the ac field is applied perpendicularly to the film surface, the calibration is done by comparing the measured low- $H_{m}$ limit of $\chi$ (the Meissner susceptibility) with accurately calculated theoretical results for completely shielded films. The theoretical $\chi$ for round films is exact. ${ }^{19}$ For square films, it has been obtained to accuracy of $0.1 \%$ by two-dimensional first-principles calculations $^{20,21}$ and by extrapolation of accurately calculated magnetometric demagnetizing factor of square bars. ${ }^{22}$

We have measured $T, f$, and $H_{m}$ dependence of the ac susceptibility of a square $\mathrm{YBa}_{2} \mathrm{Cu}_{3} \mathrm{O}_{7-\delta}$ film of sides $4.00 \pm 0.01 \mathrm{~mm}$ and nominal thickness $250 \mathrm{~nm}$ using the calibrated ac susceptometer after the magnitude correction with $k=1.081$. The measured $\chi$ versus $T$ at $f=333 \mathrm{~Hz}$ and $H_{m}=80 \mathrm{~A} / \mathrm{m}$ is shown in Fig. 8. We see that $\chi^{\prime}$ is constant and $\chi^{\prime \prime}$ is 0 at $T<60 \mathrm{~K}$, which corresponds to a Meissner state. Investigating the data carefully, we find that $\chi^{\prime}$ and $\chi^{\prime \prime}$ change slightly even at low $T$, and small differences occur in the susceptibility among different values of $f$. Using $|\chi|$ $=7259$ at $T \approx 40 \mathrm{~K}$ and $f=333 \mathrm{~Hz}$ as a reference, the relative error in $|\chi|,|\chi| / 7259-1$, as a function of $T$ is plotted in Fig. 9(a) for different values of $f$ between 111 and 10000 $\mathrm{Hz}$. This error is actually $1 / k^{\prime}-1$, where $k^{\prime}$ is an additional magnitude correction factor, so that the total factor becomes $k=1.081 k^{\prime}$. The measured $-\phi$ as a function of $T$ for different values of $f$ between 111 and $10000 \mathrm{~Hz}$ is plotted in Fig. 9(b). Since $\chi^{\prime \prime}=0$ should occur in the Meissner state, this $-\phi$ is actually $\Delta \phi$ used for the phase correction. We should mention that the above reference $|\chi|=7259$ departs from the theoretical $|\chi|=7275$ for completely shielded square film only by $-0.2 \%$.

For high fields, a paramagnetic Pd sample is provided by Quantum Design to calibrate superconducting quantum interference device magnetometers, whose mass susceptibility is $5.25 \times 10^{-6} \mathrm{~cm}^{3} / \mathrm{g}$ at $T=298 \mathrm{~K}$ with uncertainty less than $0.5 \% .{ }^{15}$ A paramagnetic $\mathrm{Dy}_{2} \mathrm{O}_{3}$ sample provided by Quantum Design has one order of magnitude higher moment susceptibility (moment per unit field) and can be used in a wide field 


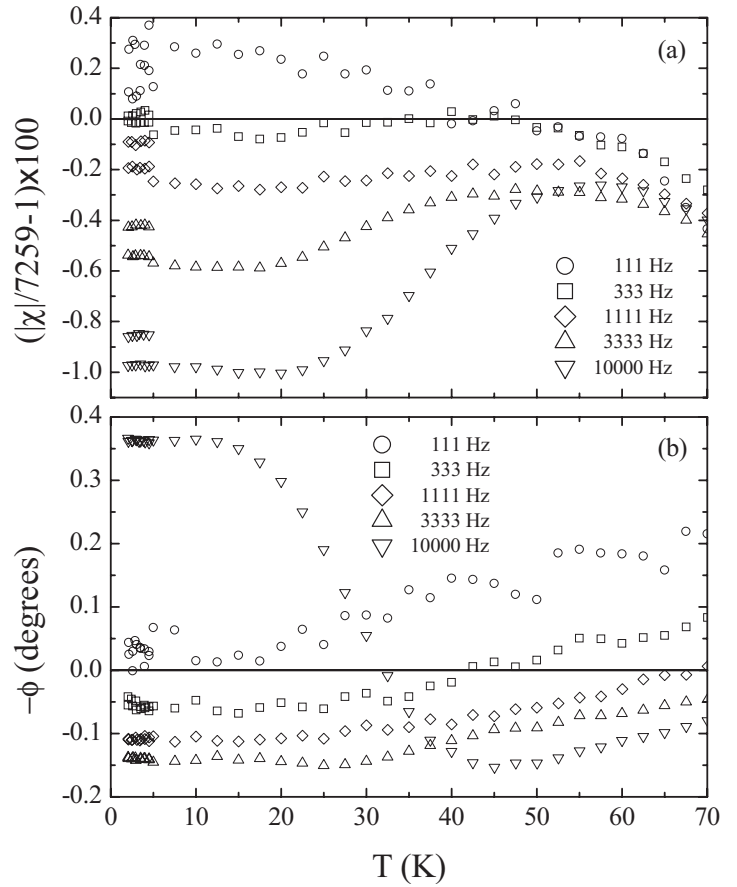

FIG. 9. The $|\chi|$ (a) and $-\phi$ (b) obtained from ac susceptibility measurements of the square superconducting film.

range. According to the Quantum Design manual, the susceptometer is calibrated at the factory using a paramagnetic $\mathrm{Dy}_{2} \mathrm{O}_{3}$ standard sample at $T=298 \mathrm{~K}, H_{m}=800 \mathrm{~A} / \mathrm{m}$, and $f$ $=1000 \mathrm{~Hz}$. The susceptibility of the standard sample is obtained by dc magnetometric measurement at $T=298 \mathrm{~K}$ and $H=800 \mathrm{kA} / \mathrm{m}$, and the dc magnetometer itself is calibrated by using a $\mathrm{Pd}$ standard sample at $T=298 \mathrm{~K}$ and $H$ $=1600 \mathrm{kA} / \mathrm{m}$. After the calibration by using copper cylinder, a calibration by using the $\mathrm{Dy}_{2} \mathrm{O}_{3}$ sample has resulted in a magnitude correction factor $k=1.080$, only $0.1 \%$ different from the value given above. The agreement between both calibrations is again perfect.

From the above facts, the accuracy of $k$ calibrated by the three different techniques seems all to be $0.2 \%$, although independent error analysis will give a larger error for each case. The accuracy by using $\mathrm{Dy}_{2} \mathrm{O}_{3}$ sample is limited by the uncertainty $0.5 \%$ of the mass susceptibility of Pd. Measuring the mass of the copper cylinder, a density of $8.96 \mathrm{~g} / \mathrm{cm}^{3}$ is obtained, which is $0.2 \%$ larger than its standard value $8.94 \mathrm{~g} / \mathrm{cm}^{3}$. The calculated eddy current $\chi\left(\theta^{2}\right)$ also has an error less than $0.2 \%$. Therefore, the $k$ calibrated by copper cylinder should have an error about $0.5 \%$. Similar problems occur when using the superconducting film. Thus, the accuracy of $k$ may be safely estimated as $0.5 \%$. Further improvements depend on the requirements in materials research, and accurate sample size determination is crucial when the susceptibility of standard samples is calculated from their sizes.

It is interesting to notice the difference in the results between the copper cylinder and the superconducting film shown in Figs. 6(c) and 9(a); the $k^{\prime}$ at $T=10 \mathrm{~K}$ obtained from the former and latter is about 1.015 and 1.01, respectively. Since 1.01 obtained directly from the Meissner effect is more reliable, there will be an error of $0.5 \%$ involved in the $k^{\prime}$ determined from the copper measurements. This error may arise from the nonuniformity in the copper cylinder owing to sample preparation and oxidation. If its surface conductivity is lower than inside, then its $|\chi|$ measured at high $f$ will be decreased owing to a weakened skin effect. Another interesting phenomenon is the very large and $T$ dependent $\Delta \phi$ determined from the copper cylinder at $f=11 \mathrm{~Hz}$ and high $T$, as shown in Fig. 5(a). Such a large $\Delta \phi$ is not found in the measurements of $\mathrm{Dy}_{2} \mathrm{O}_{3}$ sample. The difference between both is the values of $-\phi$ itself. It seems that a large error will occur at low $f$ if $-\phi$ is near $90^{\circ}$ but not if $-\phi$ is small. Its origin might be related to the error in the phase lock-in detector.

It should be mentioned that the paramagnetic $\mathrm{Dy}_{2} \mathrm{O}_{3}$ sample used in conventional calibrations is a secondary standard, which is calibrated by using another paramagnetic Pd sample as primary standard. Cooper cylinder can be used as a primary standard to calibrate ac susceptometer; and its dimensions are the only quantities that have to be known for the calibration.

\section{Negative $\chi^{\prime \prime}$ and other erroneous results}

Negative $\chi^{\prime \prime}$ and other qualitatively erroneous results may result from the phase error $-\Delta \phi(f, T)$. According to Eqs. (13)-(16), the following errors will occur if no phase correction is made. When the phase error $-\Delta \phi(f, T)<0, \chi^{\prime}$ has a positive error for both $m^{\prime}>0$ and $<0$, and $\chi^{\prime \prime}$ has a negative and positive error for $m^{\prime}>0$ and $<0$, respectively. If the phase error changes into positive, the signs of the errors in $\chi^{\prime}$ and $\chi^{\prime \prime}$ will also change. We have found all such cases in materials research.

One year before the discovery of high-temperature superconductors, a homemade ac susceptometer in the laboratory, where one of us worked, was mainly used for the study of spin glasses. With a conventional coaxial coil arrangement, the coil former was made of copper with a longitudinal slit to improve temperature uniformity and reduce eddycurrent effects. Using this ac susceptometer, several samples of rapidly quenched materials, which showed a spin glass behavior according to the literature, were measured with results qualitatively consistent with those already published. However, the spin glass like behavior $\left(\chi^{\prime}\right.$ decreases with decreasing $T$ accompanied by an increase in $\chi^{\prime \prime}$ ) was later found to occur for many other rapidly quenched materials and even for a piece of iron, and it was found for a long sample of lead, whose $\chi^{\prime}$ and $\chi^{\prime \prime}$ should be about -1 and zero, respectively, below superconducting transition temperature $T_{c}$, that with decreasing $T$ to below $T_{c},{\underset{\chi}{\prime}}^{\prime}$ and $\chi^{\prime \prime}$ change from 0 to -0.85 and -0.22 , respectively. ${ }^{23}$

Since the conductivity of copper increases with decreasing $T$, these results indicate a serious eddy-current effect on the $\chi$ measured by this ac susceptometer. In this case, the low- $T$ eddy-current effect may be described by $-\Delta \phi(f, T)$ $>0$, which causes an over-large and over-small $\chi^{\prime \prime}$ for ferromagnetic and superconducting samples, respectively, accompanied by $k(f, T)<1$, which causes a low- $T$ decrease in the magnitude of both $\chi^{\prime}$ and $\chi^{\prime \prime}$.

Similar phase error occurred in the homemade high-field ac susceptometer mentioned above, working at $77 \mathrm{~K}^{12,14}$ 

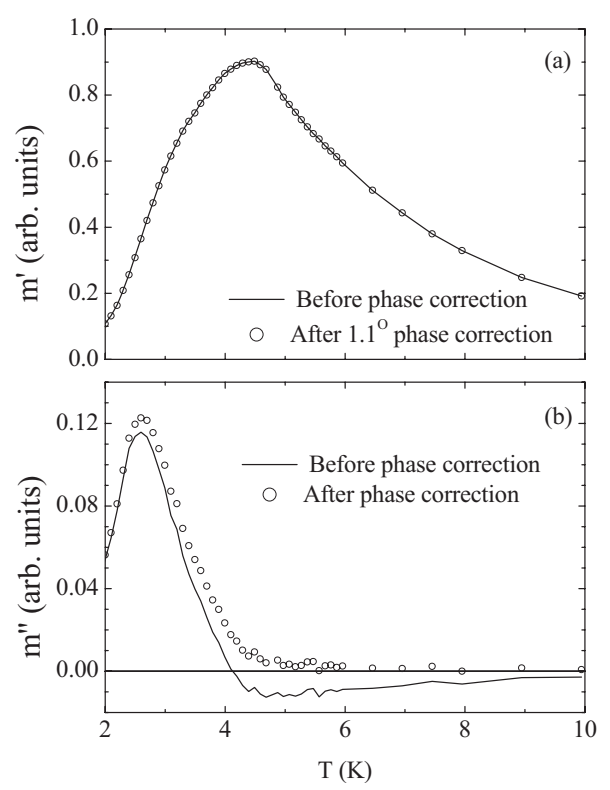

FIG. 10. Directly recorded (lines) $m^{\prime}$ and $m^{\prime \prime}$ as functions of $T$ for a molecular magnetic sample measured using a PPMS ac susceptometer at $f$ $=11 \mathrm{~Hz}, H_{\mathrm{dc}}=40 \mathrm{kA} / \mathrm{m}$, and $H_{m}=280 \mathrm{~A} / \mathrm{m}$, compared with those after a phase correction of $\Delta \phi=1.1^{\circ}$ (symbols).

Without a phase correction, $\chi^{\prime \prime}$ of superconducting samples measured at high $f$ was always negative at low $H_{m}$, owing to the positive error in phase.

We give a new example for a sample of molecular magnet measured using another PPMS ac susceptometer. The $m^{\prime}$ and $m^{\prime \prime}$ of the sample measured at $f=11 \mathrm{~Hz}, H_{\mathrm{dc}}$ $=40 \mathrm{kA} / \mathrm{m}$, and $H_{m}=280 \mathrm{~A} / \mathrm{m}$ are plotted by solid lines in Fig. 10 as functions of $T$. We see that $m^{\prime \prime}<0$ occurs at $T$ $>4 \mathrm{~K}$. The symbols are the results after a phase correction by using Eqs. (13) and (14) with $\Delta \phi=1.1^{\circ}$, obtained by a calibration as done above. It is clear that $m^{\prime \prime} \geq 0$ occurs after the correction.

A negative $\chi^{\prime \prime}$ has been repeatedly discovered in the literature. ${ }^{6-10}$ Although such results are unphysical, it is still interesting to study each case, so that the ac susceptibility technique can be improved. Careful calibration using proper standard samples is necessary for such a study.

\section{E. Materials research using homemade ac susceptometers calibrated with copper cylinders}

The discovery of negative $\chi^{\prime \prime}$ and other erroneous results had led to innovation and improvements of a homemade low $T$ ac susceptometer mentioned above, as described in detail in Ref. 23. The innovated ac susceptometer had a coaxial and concentric coil assembly, Teflon coil formers, a specially configurated thermocouple temperature sensor, and a glass Dewar. After a calibration with a copper cylinder and relevant corrections, this susceptometer was used for measuring $\chi\left(f, T, H_{m}\right)$ of various materials.

Instead of a well machined copper cylinder with aspect ratio being 1, the copper cylinder was cut from a copper wire of diameter $2.67 \mathrm{~mm}$, and analytical formulas for ac susceptibility of infinitely long conducting cylinder were used to calculate its ac susceptibility followed by a demagnetizing correction. The results were very similar to the present improved case. The accuracy of demagnetizing correction was later justified in Ref. 14.

Using the improved susceptometer, typical spin glass properties of $\mathrm{Ni}_{89} \mathrm{Cr}_{11}$ and $\mathrm{Gd}_{4} \mathrm{Al}_{3}$ were measured and reported in Ref. 23. The cold rolling effect on magnetic transition of $\mathrm{Au}_{83} \mathrm{Fe}_{17}$ was studied in Ref. 24. Magnetic transitions of single crystal Er were studied in Ref. 25. Using another homemade ac susceptometer with essentially the same design but a metallic Dewar, magnetic transitions of single crystal $\mathrm{Nd}_{2} \mathrm{Fe}_{14} \mathrm{~B}$ were studied. ${ }^{26}$ It was shown that both homemade ac susceptometers had similar performance to the LakeShore ac susceptometer, which was used in a later study of aligned epoxy-bound $\mathrm{Sm}_{2} \mathrm{Fe}_{17}{ }^{27}$

Some work on newly discovered high-temperature superconductors was carried out using the improved ac susceptometer, with results confirmed later by others. ${ }^{28-31}$ The $\chi(T)$ of sintered and powdered $\mathrm{YBa}_{2} \mathrm{Cu}_{3} \mathrm{O}_{x}$ high-temperature superconductor were shown in Ref. 32, leading to the identification of the intergranular and intragranular critical-current density $J_{c}$. The field dependent intergranular $J_{c}$ was then determined by $\chi\left(H_{m}, T\right)$ measurements based on a critical-state model calculation. ${ }^{33,34}$ Treating the $\chi\left(H_{m}, T\right)$ data measured by this susceptometer, the strong link between $\mathrm{Bi}_{2} \mathrm{Sr}_{2} \mathrm{Ca}_{2} \mathrm{Cu}_{3} \mathrm{O}_{y}$ grains $\left(J_{c} \approx 10^{4} \mathrm{~A} / \mathrm{cm}^{2}\right.$ at $\left.T=97 \mathrm{~K}\right)$ was predicted. ${ }^{35}$

Research later concentrated on $\chi\left(H_{m}, f\right)$ of sintered hightemperature superconductors at $T=77 \mathrm{~K}$. In this case, since the entire coil assembly is immersed in liquid nitrogen, $H_{m}$ may be easily increased to $3 \times 10^{4} \mathrm{~A} / \mathrm{m}$, so that both the intergranular $J_{c}$ and the properties of the grains can be studied. ${ }^{36-38}$ For tapes, films, rings, and disks with high $J_{c}$, the high-field ac susceptometer mentioned above ${ }^{12}$ allows $H_{m}$ to be as large as $10^{5} \mathrm{~A} / \mathrm{m}^{39-45}$

\section{F. Model calculations}

Model calculations with accuracy better than $0.1 \%$ are essential for the calibration of ac susceptometers. As a summary, such calculations with accuracy about $0.1 \%$ have been carried out for the magnetometric demagnetizing factor of cylinders, square bars, and rectangular prisms, ${ }^{16,17,22,46,47}$ for the ac susceptibility of conducting cylinders and magnetic conducting spheres, ${ }^{14,18}$ and for the perpendicular susceptibility of completely shielded rectangular films. ${ }^{20,21}$

Using well calibrated ac susceptometers to study superconducting materials, model calculations (with lower accuracy) for the properties of the samples are important. Our attention has been concentrated in the critical-state model, flux creep model, and flux flow model calculations. ${ }^{33,41,48-54}$

The present work concentrates on the accuracy of $\chi$ measurements. Another problem that has not been considered seriously is how to convert the accurately measured $\chi$ of the sample into the $\chi$ of the studied material. For paramagnetic or superparamagnetic materials, this problem has been solved theoretically by a proper demagnetizing correction. ${ }^{22}$ It should also be studied experimentally. 


\section{CONCLUSIONS}

The principle and procedures of the calibration and correction of low-temperature ac susceptometers using a copper cylinder as the standard sample are described. This calibration is independent, without requiring another primary standard sample, and its accuracy is determined by the accuracy of the copper cylinder sizes and its theoretical susceptibility. It can be used for all kinds of ac susceptometers, especially for those working at low temperatures, to greatly improve their performance. Recommendations are made for solving the long-term problem of frequently reported anomalous results, like negative $\chi^{\prime \prime}$.

\section{ACKNOWLEDGMENTS}

The authors are grateful to R. B. Goldfarb for his continuous interest in and kind support to the work described in this paper, to Thomas Reed and A. Palau for preparing and providing the copper cylinder and superconducting film, to N. Domingo for providing the original data of Fig. 10, and to B. Martinez and B. Bozzo for support and help in the experiments. Financial support from Consolider Project Nos. CSD2007-00041 and 2009SGR-1292 is acknowledged.

${ }^{1}$ A. J. van Duyneveldt, J. Appl. Phys. 53, 8006 (1982).

${ }^{2}$ A. J. van Duyneveldt, Proceedings of Letnia Szkola Magnetyzmu, Bialowieza, 1989, p. 1.

${ }^{3}$ F. Palacio, F. J. Lazaro, and A. J. van Duyneveldt, Mol. Cryst. Liq. Cryst. 176, 289 (1989).

${ }^{4}$ C. Y. Huang, J. Magn. Magn. Mater. 51, 1 (1985).

${ }^{5}$ R. B. Goldfarb, M. Lelental, and C. A. Thompson, in Magnetic Susceptibility of Superconductors and Other Spin Systems, edited by R. A. Hein, T.

L. Francavilla, and D. H. Liebenberg (Plenum, New York, 1991), p. 49.

${ }^{6}$ S. N. Kaul and S. Srinath, Phys. Rev. B 62, 1114 (2000).

${ }^{7}$ E. M. Levin, V. K. Pecharsky, and K. A. Gschneidler, Jr., Phys. Rev. B 62, R14625 (2000).

${ }^{8}$ A. García-Valenzuela and R. G. Barrera, Physica B 338, 31 (2003).

${ }^{9}$ A. N. Ulyanov, H. D. Quang, K. W. Lee, S. C. Yu, N. H. Sinh, Y. M. Kang, and S. I. Yoo, IEEE Trans. Magn. 44, 3060 (2008).

${ }^{10}$ M. K. Singh, W. Prellier, M. P. Singh, R. S. Katiyar, and J. F. Scott, Phys. Rev. B 77, 144403 (2008).

${ }^{11}$ R. B. Goldfarb and J. V. Minervini, Rev. Sci. Instrum. 55, 761 (1984).

${ }^{12}$ D.-X. Chen, Meas. Sci. Technol. 15, 1195 (2004).

${ }^{13}$ Quantum Design, Physical Property Measurement System Hardware and Options, ACMS User's Manual.

${ }^{14}$ D.-X. Chen and C. Gu, IEEE Trans. Magn. 41, 2436 (2005).

${ }^{15}$ NBS Standard Reference Materials, Magnetic Gram Susceptibility, $763-$ Aluminum, 764-Platinum, 765-Palladium, 766-Manganese Fluoride (1973).

${ }^{16}$ D.-X. Chen, J. A. Brug, and R. B. Goldfarb, IEEE Trans. Magn. 27, 3601 (1991).

${ }^{17}$ D.-X. Chen, E. Pardo, and A. Sanchez, J. Magn. Magn. Mater. 306, 135 (2006).

${ }^{18}$ C. Prados, R. P. del-Real, D.-X. Chen, B.-Z. Li, and A. Hernando, Rev. Sci. Instrum. 65, 3044 (1994).

${ }^{19}$ J. R. Clem and A. Sanchez, Phys. Rev. B 50, 9355 (1994).
${ }^{20}$ D.-X. Chen, C. Navau, N. Del-Valle, and A. Sanchez, Appl. Phys. Lett. 92, 202503 (2008).

${ }^{21}$ D.-X. Chen, C. Navau, N. Del-Valle, and A. Sanchez, Physica C 469, 732 (2009).

${ }^{22}$ E. Pardo, D.-X. Chen, and A. Sanchez, IEEE Trans. Magn. 40, 1491 (2004).

${ }^{23}$ D.-X. Chen, Ballistic and Bridge Methods of Magnetic Measurements of Materials (China Metrology, Beijing, 1990), p. 526.

${ }^{24}$ D.-X. Chen, J. Nogués, K. V. Rao, C. E. Violet, and R. J. Borg, Chin. Phys. Lett. 5, 129 (1988).

${ }^{25}$ H. U. Astrom, D.-X. Chen, G. Benediktsson, and K. V. Rao, J. Phys.: Condens. Matter 2, 3349 (1990).

${ }^{26}$ D.-X. Chen, V. Skumryev, and H. Kronmüller, Phys. Rev. B 46, 3496 (1992).

${ }^{27}$ D.-X. Chen, V. Skumryev, and J. M. D. Coey, Phys. Rev. B 53, 15014 (1996).

${ }^{28}$ K. V. Rao, D.-X. Chen, J. Nogués, C. Politis, C. Gallo, and J. A. Gerber, in Proceedings of the Symposium on High-Temperature Superconductors, edited by D. U. Gubser and M. Schliter (MRS, Pittsburg, 1987), Vol. EA-11, p. 133.

${ }^{29}$ D.-X. Chen, J. Nogués, N. Karpe, and K. V. Rao, Kexue Tongbao (Foreign Lang. Ed.) 32, 1620 (1987).

${ }^{30}$ D.-X. Chen, V. Skumryev, N. Karpe, R. Puzniak, and K. V. Rao, J. Phys. (Paris) 49, 2183 (1988).

${ }^{31}$ Yu. Mei, H. L. Luo, D.-X. Chen, J. Nogués, and K. V. Rao, J. Appl. Phys. 64, 2533 (1988)

${ }^{32}$ D.-X. Chen, R. B. Goldfarb, J. Nogués, and K. V. Rao, J. Appl. Phys. 63, 980 (1988).

${ }^{33}$ D.-X. Chen and R. B. Goldfarb, J. Appl. Phys. 66, 2489 (1989).

${ }^{34}$ D.-X. Chen, J. Nogués, and K. V. Rao, Cryogenics 29, 800 (1989).

${ }^{35}$ D.-X. Chen, Y. Mei, and H. L. Luo, Physica C 167, 317 (1990).

${ }^{36}$ D.-X. Chen, A. Sanchez, T. Puig, L. M. Martinez, and J. S. Munoz, Physica C 168, 652 (1990).

${ }^{37}$ D.-X. Chen and A. Sanchez, Phys. Rev. B 45, 10793 (1992).

${ }^{38}$ D.-X. Chen, K.-Y. Wang, and A. Hernando, Physica C 274, 39 (1997).

${ }^{39}$ D.-X. Chen, E. Pardo, A. Sanchez, A. Palau, T. Puig, and X. Obradors, Appl. Phys. Lett. 85, 5646 (2004).

${ }^{40}$ D.-X. Chen, E. Pardo, A. Sanchez, S.-S. Wang, Z.-H. Han, E. Bartolome, T. Puig, and X. Obradors, Phys. Rev. B 72, 052504 (2005).

${ }^{41}$ D.-X. Chen, E. Pardo, A. Sanchez, and E. Bartolome, Appl. Phys. Lett. 89, 072501 (2006).

${ }^{42}$ D.-X. Chen, E. Pardo, A. Sanchez, M. N. Iliev, S.-S. Wang, and Z.-H. Han, J. Appl. Phys. 101, 073905 (2007).

${ }^{43}$ D.-X. Chen, E. Pardo, and A. Sanchez, Appl. Phys. Lett. 91, 012506 (2007).

${ }^{44}$ D.-X. Chen, E. Bartolome, E. Pardo, A. Sanchez, B. Bozzo, X. Granados, T. Puig, X. Obradors, and H. Claus, Physica C 460-462, 770 (2007).

${ }^{45}$ D.-X. Chen, A. Sanchez, C. Navau, Y.-H. Shi, and D. A. Cardwell, Supercond. Sci. Technol. 21, 085013 (2008).

${ }^{46}$ E. Pardo, D.-X. Chen, and A. Sanchez, J. Appl. Phys. 96, 5365 (2004).

${ }^{47}$ D.-X. Chen, E. Pardo, and A. Sanchez, IEEE Trans. Magn. 41, 2077 (2005).

${ }^{48}$ D.-X. Chen, A. Sanchez, and J. S. Munoz, J. Appl. Phys. 67, 3430 (1990).

${ }^{49}$ D.-X. Chen and A. Sanchez, J. Appl. Phys. 70, 5463 (1991).

${ }^{50}$ E. Pardo, D.-X. Chen, A. Sanchez, and C. Navau, Supercond. Sci. Technol. 17, 537 (2004).

${ }^{51}$ D.-X. Chen, E. Pardo, and A. Sanchez, Supercond. Sci. Technol. 18, 997 (2005).

${ }^{52}$ D.-X. Chen, E. Pardo, and C. Gu, Supercond. Sci. Technol. 18, 1280 (2005).

${ }^{53}$ D.-X. Chen and E. Pardo, Appl. Phys. Lett. 88, 222505 (2006).

${ }^{54}$ D.-X. Chen, C. Navau, N. Del-Valle, and A. Sanchez, Physica C 470, 89 (2010). 